Research Article

\title{
Strength Investigation of the Silt-Based Cemented Paste Backfill Using Lab Experiments and Deep Neural Network
}

\author{
Chongchun Xiao, ${ }^{1,2}$ Xinmin Wang, ${ }^{1}$ Qiusong Chen $\mathbb{D}^{1,3}$ Feng Bin, ${ }^{2}$ Yihan Wang, \\ and Wei Wei ${ }^{1,5}$ \\ ${ }^{1}$ School of Resources and Safety Engineering, Central South University, Changsha 410083, China \\ ${ }^{2}$ Feny Co. Ltd., Changsha 410083, China \\ ${ }^{3}$ Sinosteel Maanshan Institute of Mining Research Company Limited, Maanshan 243000, China \\ ${ }^{4}$ Hunan Filling Engineering Technology Co. Ltd., Changsha 410083, China \\ ${ }^{5}$ Wanbao Mining Ltd., Beijing 100000, China \\ Correspondence should be addressed to Qiusong Chen; qiusong.chen@csu.edu.cn
}

Received 5 November 2020; Revised 21 November 2020; Accepted 8 December 2020; Published 24 December 2020

Academic Editor: Erol Yilmaz

Copyright (C) 2020 Chongchun Xiao et al. This is an open access article distributed under the Creative Commons Attribution License, which permits unrestricted use, distribution, and reproduction in any medium, provided the original work is properly cited.

\begin{abstract}
The cemented paste backfill (CPB) technology has been successfully used for the recycling of mine tailings all around the world. However, its application in coal mines is limited due to the lack of mine tailings that can work as aggregates. In this work, the feasibility of using silts from the Yellow River silts (YRS) as aggregates in CPB was investigated. Cementitious materials were selected to be the ordinary Portland cement (OPC), OPC + coal gangue (CG), and OPC + coal fly ash (CFA). A large number of lab experiments were conducted to investigate the unconfined compressive strength (UCS) of CPB samples. After the discussion of the experimental results, a dataset was prepared after data collection and processing. Deep neural network (DNN) was employed to predict the UCS of CPB from its influencing variables, namely, the proportion of OPC, CG, CFA, and YS, the solids content, and the curing time. The results show the following: (i) The solid content, cement content (cement/sand ratio), and curing time present positive correlation with UCS. The CG can be used as a kind of OPC substitute, while adding CFA increases the UCS of CPB significantly. (ii) The optimum training set size was $80 \%$ and the number of runs was 36 to obtain the converged results. (iii) GA was efficient at the DNN architecture tuning with the optimum DNN architecture being found at the 17th iteration. (iv) The optimum DNN had an excellent performance on the UCS prediction of silt-based CPB (correlation coefficient was 0.97 on the training set and 0.99 on the testing set). (v) The curing time, the CFA proportion, and the solids content were the most significant input variables for the silt-based $\mathrm{CPB}$ and all of them were positively correlated with the UCS.
\end{abstract}

\section{Introduction}

Mining and minerals industries are very likely to cause widespread environmental damage, such as noise pollution, air and water contamination, and solids waste generation $[1,2]$. Thus, recycling has been an important issue encountered by mining and minerals industries, especially the solids waste recycling [3-6]. As for the metal mining, solid waste recycling mainly deals with the large amount of mine tailings generated due to the high waste-to-product ratio $[7,8]$. It has been shown that the waste-to-product ratio for the metal mining is often $100: 1$ in volume, which can be as high as 1,000,000:1 in some cases [9]. Interested readers could refer to the review paper in [7] for a detailed presentation of the tailings generated for typical ores.

In the era of sustainable development and cleaner production, the recycling of mine tailings has been a hot topic that has undergone extensive investigations. A number of recycling methods have been proposed, among which the cemented paste backfill $(\mathrm{CPB})$ is one of the most popular methods. $\mathrm{CPB}$ is advantageous in that it can not only promote the tailings recycling, but also improve working 
conditions, stabilise surrounding rock mass, and sometimes work as the platform for the subsequent mining operations. Therefore, CPB has been widely used throughout the world, such as in China [10-13], Australia [14], Canada [15], and Turkey $[16,17]$, to name a few.

Depending on the intended function of the backfill, a certain amount of mechanical properties, often indicated by unconfined compressive strength (UCS), is needed for CPB. For example, a UCS between $150 \mathrm{kPa}$ and $300 \mathrm{kPa}$ is required to reduce the liquefaction possibility while a UCS larger than $4 \mathrm{MPa}$ is recommended by Grice when CPB is used for roof support [7]. The determination of UCS is essential prior to any engineering application of the CPB technology. Traditionally, lots of lab experiments are conducted for each mine site, which consumes a large amount of time, materials, and human resources. The strength prediction from its influencing variables is attracting increasing attention from both academia and industry. A large number of studies have been performed by Qi and his co-authors [18-20], followed by the most up-to-date papers like [21-23].

Up to now, the advantages of CPB, the importance of UCS, and the significance of strength prediction have been presented mainly for metal mining. The application of CPB in coal mines has also been investigated. For example, Huang et al. [24] proposed a technological framework for the backfill technology that used coal waste and fly ash as the main ingredients. Zhang et al. [25] investigated the surface subsidence in backfill coal mining based on the equivalent mining height theory. In contrast to the CPB technology in metal mining that recycles mine tailings, the application of the CPB technology in coal mining requires the selection of aggregates. In some cases, the lack of suitable aggregates limits the application of $\mathrm{CPB}$, thus hindering the cleaner production in coal mining.

In this work, the strength development of silt-based CPB was investigated using lab experiments and deep neural network (DNN). The main contributions of this work to the literature are as follows:

(i) To facilitate the application of CPB in coal mines, the utilization of Yellow River silts (YRS) as aggregates in $\mathrm{CPB}$ was investigated

(ii) The comparison of ordinary Portland cement (OPC), OPC + coal gangue (CG), and OPC + coal fly ash (CFA) as the cementitious materials was conducted

(iii) A DNN model was proposed for the strength prediction of the silt-based $\mathrm{CPB}$

\section{Study Site}

A coal mine (referred to as 'A-Mine'), located in Shandong Province, China, is a medium-sized coal mine with the production of 900,000 tonnes per year. As an underground coal mine, the earth surface above the mined-out area is covered with town and farmland, and the CPB technology has thus become legally required. To realize the engineering application of $\mathrm{CPB}$, the first work is choosing the backfill aggregate and cementitious materials, which affect the mechanical properties of CPB. The principals of selecting backfill materials include extensive source, low cost, pollution free, etc. Considering the location of A-Mine that is nearby the Yellow River and a thermal power plant, as shown in Figure 1, the silt from Yellow River (YRS), CG, and CFA were considered as potential materials for $\mathrm{CPB}$.

\section{Materials and Methods}

3.1. Materials. The YRS has been analyzed as a potential backfill aggregate, which was selected from a silts plant near A-Mine. The main cementitious material was No. 42.5 ordinary Portland cement (OPC, according to Chinese National Standard, GB175-2007) provided by A-Mine. In order to improve the mechanical and pipe transport properties of $\mathrm{CPB}$, other materials, CFA and CG, were also used for modification. The CFA was collected from a thermal power plant, as shown in Figure 1, while the CG was collected from a CG dump belonging to A-Mine. All these materials were collected and sealed and then sent to Central South University for test. The underground water from A-Mine was used for preparing the CPB samples. It should be noted that the YRS needs to be pretreated to remove the impurities, and the CG particles were controlled in $5 \mathrm{~mm}$, as presented in Figure 2.

The particles size distributions of YRS and CG were measured by sieving method and laser particle size analyzer (Malvern Mastersizer 2000), as shown in Figure 3, and Table 1 summarizes the main PSD parameters. The mineral components were tested using X-ray diffraction (XRD; Bruker AXS D8 Advance Diffractometer), as shown in Figure 4. The results show that the median particle size of silts and CG was $145 \mu \mathrm{m}$ and $380 \mu \mathrm{m}$, which can be classified as coarse aggregates [26]. Table 2 presents the chemical properties of YRS and CG. The main mineral components of the YRS are quartz, feldspar, and calcite. Among them, the quartz and feldspar have been proven important for the strength of $\mathrm{CPB}$. The kaolinite, quartz, and calcite gypsum are the main components of CG, which are good for improving the mechanical properties of $\mathrm{CPB}$.

\subsection{Lab Experiments}

3.2.1. CPB Preparation. The backfill materials, i.e., YRS, CG, CFA, OPC, water, etc., were measured as designed and then mixed using an electric mixer (JJ-5, Hebei, China). After being mixed for $5 \mathrm{~min}$, the uniform CPB slurry was formed and poured into triple plastic moulds $(7.07 \times 7.07 \times 7.07 \mathrm{~cm})$. The moulds should be poured with CPB slurry as much as possible, to avoid the effects of consolidation shrinkage [27], as shown in Figure 5(a). After 24 hours and CPB initial setting, the samples were scraped to form a smooth surface (Figure 5)(b), and the moulds were removed. The cubic CPB samples (Figure 5)(c) were then cured in a curing box (YH-40B, Qingda, Tianjin of China) with the temperature of $25^{\circ} \mathrm{C}$ and humidity of $90 \%$ (Figure $5(\mathrm{~d})$ ).

3.2.2. UCS Test. The CPB samples after curing for $1 \mathrm{~d}, 3 \mathrm{~d}$, and $28 \mathrm{~d}$ were taken out for UCS tests using pressure testing machine (WDW-20), as shown in Figure 6. The test sample 


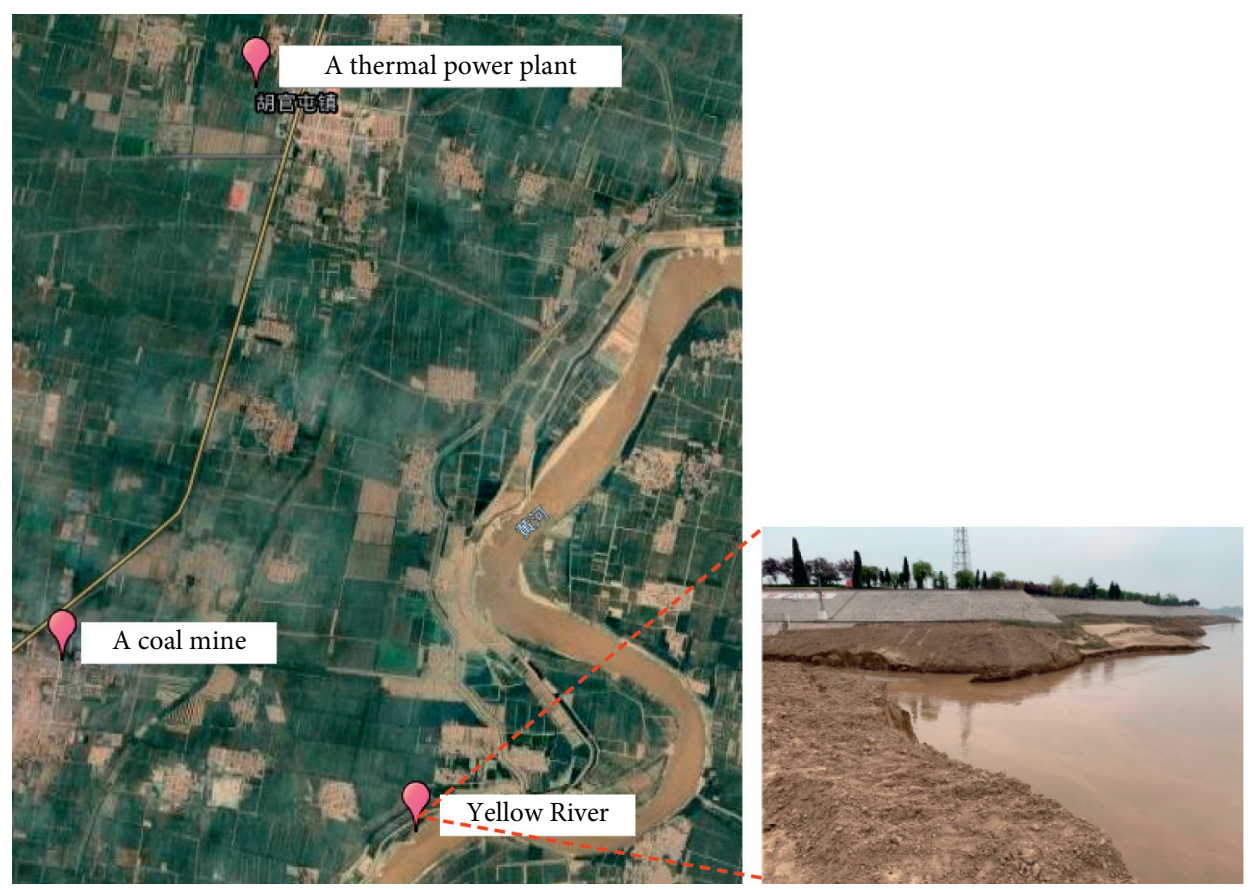

FIgURe 1: The location of study site.

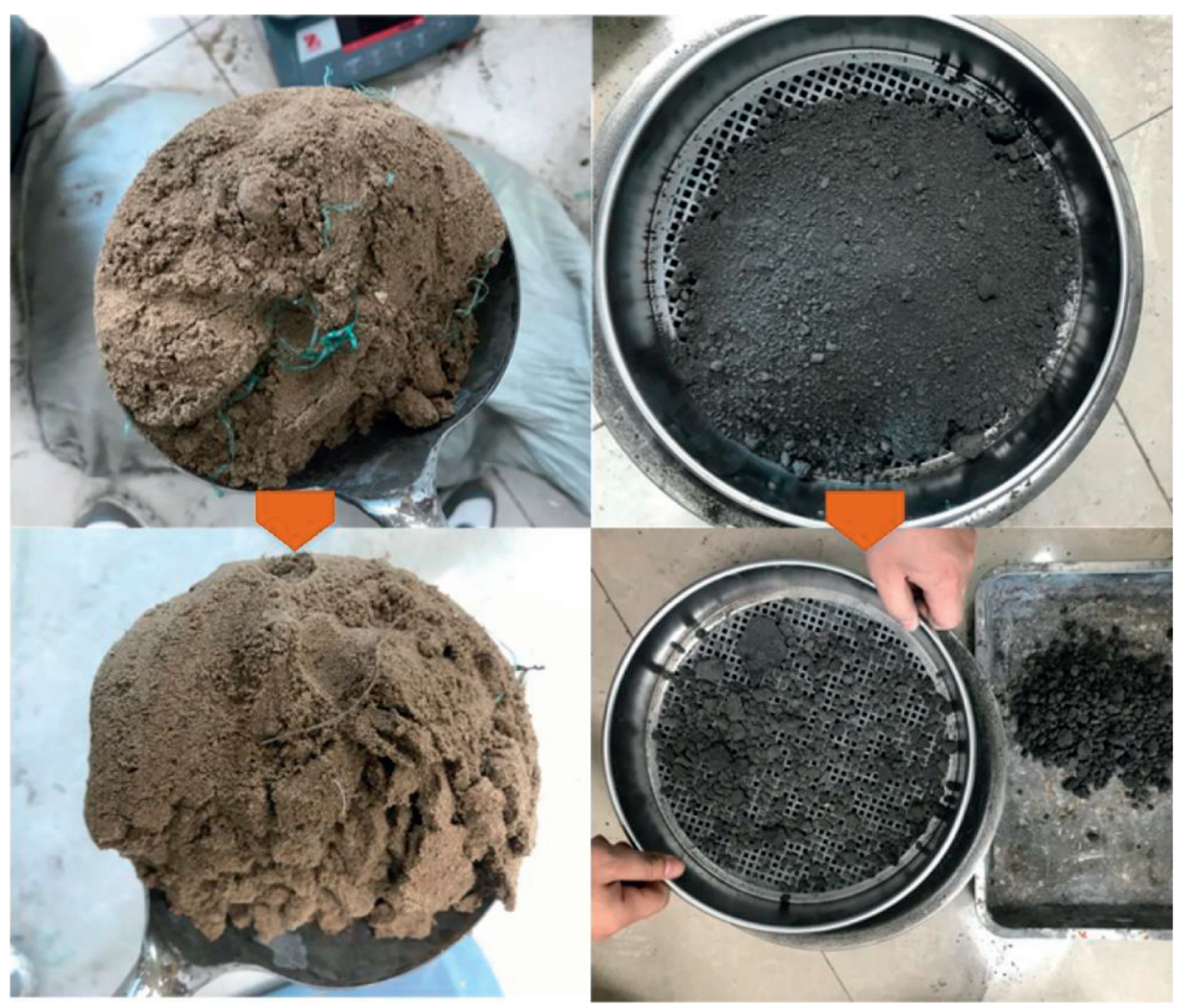

FIGURE 2: YRS pretreatment and CG sieving.

was placed in the circular testing plate and tested with a loading speed of $0.5 \mathrm{~mm} / \mathrm{min}$ [28]. Three samples were tested in each group of experiments, and the average value of the two results with errors less than $5 \%$ was taken as UCS.
3.3. Deep Neural Network Prediction. Artificial neural network (ANN) is a computing technique inspired by the biological neural networks in brain. ANN is designed to learn the mapping from inputs to outputs with a large number of 


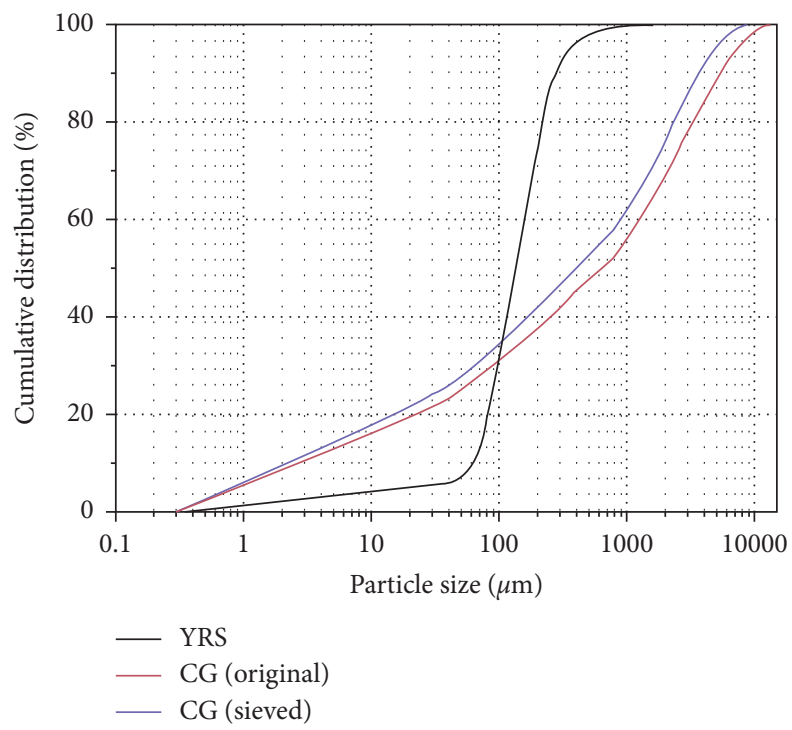

FIgURe 3: Particle size distribution curve of aggregate.

TABLE 1: Main geotechnical properties of aggregate.

\begin{tabular}{lccccccc}
\hline Aggregate & Specific gravity $(\mathrm{GS})\left(\mathrm{t} / \mathrm{m}^{3}\right)$ & $d_{60}(\mu \mathrm{m})$ & $d_{50}(\mu \mathrm{m})$ & $d_{30}(\mu \mathrm{m})$ & $d_{10}(\mu \mathrm{m})$ & Uniformity coefficient $\left(C_{u}\right)$ & Curvature coefficient $\left(C_{c}\right)$ \\
\hline YRS & 2.57 & 165 & 145 & 100 & 50 & 3.3 & 1.21 \\
CG (sieved) & 2.53 & 890 & 380 & 62 & 2.2 & 404.5 & 1.96 \\
\hline
\end{tabular}

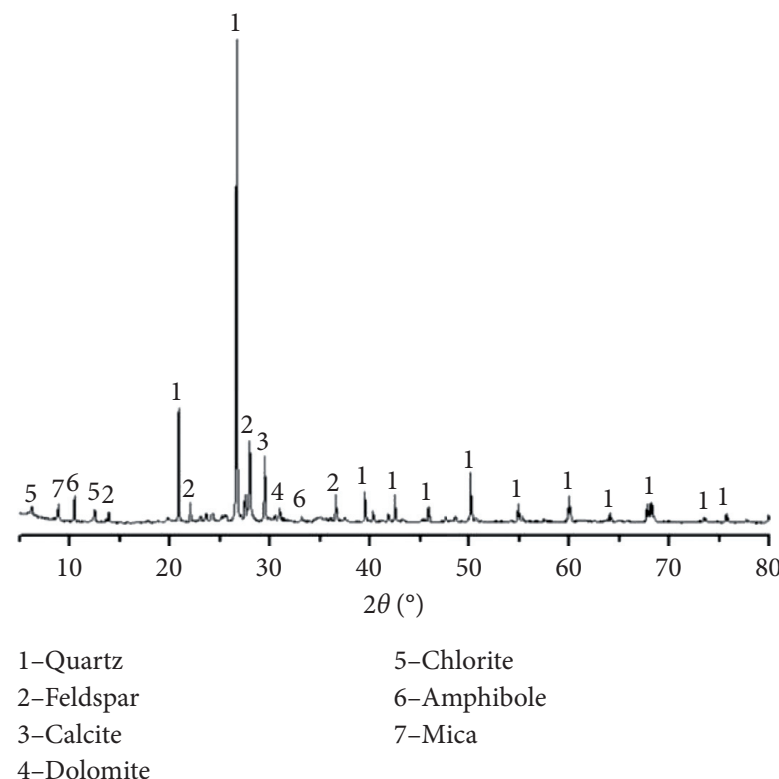

(a)

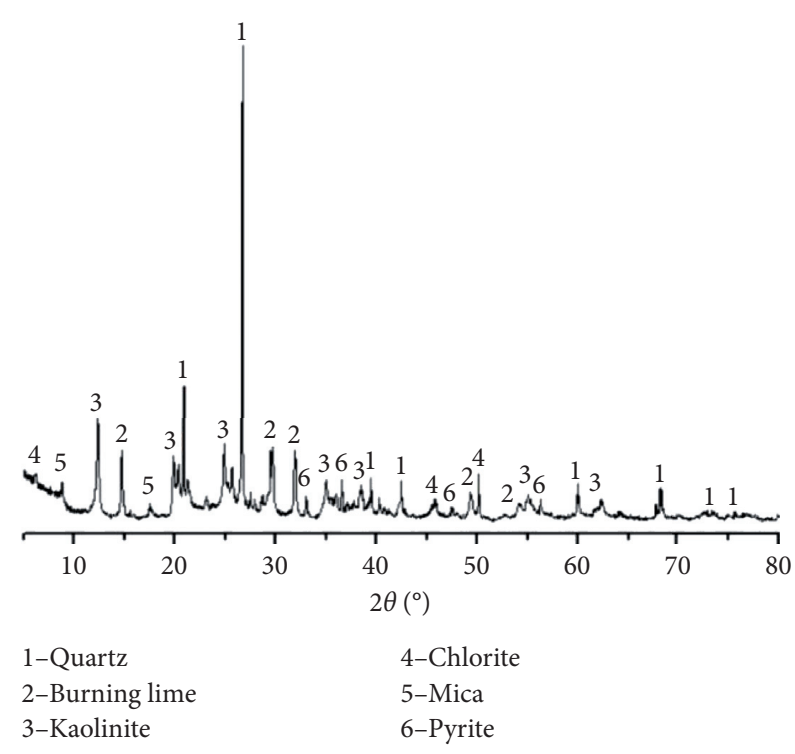

(b)

FIGURE 4: XRD mineralogical composition analysis results of aggregate: (a) YRS and (b) CG.

connected neurons, or processing units. Neurons are made up of input units and output units, where input units receive information from the previous layer and, after proper processing, output such information to the next layer. DNN is a type of ANN that has multiple hidden layers, usually larger than 3. Interested readers about ANN could refer to [29] for a detailed explanation of ANN. In this work, DNN with 5 hidden layers was used as the prediction method [30]. 
TABLE 2: The chemical properties of YS and CG (\%).

\begin{tabular}{|c|c|c|c|c|c|c|c|}
\hline Composition & Quartz & Feldspar & Calcite & Dolomite & Chlorite & Amphibole & Mica \\
\hline YRS & 32.73 & 31.29 & 14.73 & 3.27 & 5.82 & 5.29 & 6.87 \\
\hline CG & 25.01 & 15.51 & 10.31 & 4.56 & 4.89 & 5.22 & 34.50 \\
\hline
\end{tabular}

Except the number of neurons in each hidden layer, which are tuned by genetic algorithm, other DNN parameters were selected to be the default values from Sklearn [31].

The experimental results were collected for the preparation of the dataset. In this work, the proportion of OPC, CG, CFA, and silts, the solids content, and the curing time were selected to be the inputs while the UCS was selected to be the output. A total number of 126 data samples were included in the dataset. Data normalisation was performed to speed up the DNN calculations and improve its performance. The correlation coefficient, which ranged from -1 to 1 , was used as the performance indicator.

In supervised learning, the whole dataset needs to be split into the training set (for DNN training) and the testing set (for DNN verification). However, the training set size has a significant influence on the DNN performance and thus needs to be properly determined. A convergence test was conducted in this work where the training set size was increased from $30 \%$ to $90 \%$. Note that the default ANN structure from [31] was used during the convergence test for the training set size. Moreover, each training set size was repeated 50 times to obtain the converged results. The training performance was determined by 5 -fold cross-validation. As for the testing performance, the whole training set was used to train the ANN model and the trained ANN model was used to make a prediction on the testing set.

Figure 7 illustrates the results of the convergence test. As shown, the training performance increased with the increasing training set size. In contrast, the testing performance increased when the training set size was increased from $30 \%$ to $80 \%$. Then, the testing performance decreased when the training set size was further increased to $90 \%$. Therefore, the training set size was determined to be $80 \%$ in the following calculations.

As indicated before, 50 runs were repeated for each training set size to obtain the converged results. However, 50 runs might not be necessary. Moreover, it would be too computationally intensive if 50 runs were repeated for each DNN architecture during the tuning of the number of neurons in each hidden layer. Thus, a convergence test was also conducted to determine the number of runs needed for the converged results. Figure 8 shows the influence of the number of runs on the average correlation coefficient and its standard deviation. It was found that the average correlation coefficient and its standard deviation stabilized after 36 runs. Therefore, the number of runs was determined to be 36 in the following calculations.

The employed DNN had one input layer with 6 neurons, one output layer with 1 neuron, and 5 hidden layers. The number of neurons in each hidden layer would affect the DNN performance, as indicated in many previous studies $[18,30]$. In this work, the number of neurons was determined for each hidden layer within the range of 1 to 200 .
Genetic algorithm (GA) was used for the tuning of the number of neurons in each hidden layer, which was equivalent to the optimization of the DNN architecture. During the GA optimization, each DNN structure would be represented by a chromosome. The average training performance of the DNN architecture was used as the objective function for GA, which would be maximized during GA iterations.

In this study, the number of chromosomes in GA was selected to be 100 and the maximum generation was 50 . Tournament selection was utilized to select the chromosomes that will be maintained in the next generation. The crossover probability and the mutation probability were selected to be 0.90 and 0.02 , respectively. The authors note that the selection of GA parameters was based on the recommendations from the literature [32]. Interested readers could refer to [30] for a detailed discussion about the optimization of ANN architecture using GA, which was not covered in detail for clarity purpose.

\section{Results and Discussion}

4.1. Strength Development of $C P B$. Figure 9 presents the relation of UCS and the solid content of CPB at the cement/ sand ratio of $1: 8$. It can be seen that the UCS values increase with the increase of solid content. Take YRS group, for example; the UCS values of CPB samples curing for 28 days at the solid content of $73 \%, 74 \%$, and $75 \%$ are $0.73 \mathrm{MPa}$, $0.79 \mathrm{MPa}$, and $0.88 \mathrm{MPa}$, respectively. As studied before, the hardened $\mathrm{CPB}$ is an inhomogeneous body composed of crystals, gels, incompletely hydrated cement particles, free water, and pores. Among them, the crystals and gels play a role in increasing strength, while the free water leads to the decrease of strength [33]. Thus, increasing the solid content leads to the reduction of free water in the $\mathrm{CPB}$ mixture, which will increase the strength of the $\mathrm{CPB}$ thereby.

Figure 10 presents the relation of UCS and cement/sand ratio at the solid content of $74 \%$. It can be seen that increasing the cement/sand ratio significantly improves the UCS values, especially for the CPB samples curing for 28 days. Take YRS group, for instance; the UCS values of CPB curing for 28 days at the cement/sand ratio of $1: 15,1: 10,1$ : 8, and $1: 6$ are $0.17 \mathrm{MPa}, 0.51 \mathrm{MPa}, 0.79 \mathrm{MPa}$, and $1.30 \mathrm{MPa}$, respectively. It is well known that the hydration of cement provides crystals and gels in the $\mathrm{CPB}$ mixture, which is the essential reason for hardening the $\mathrm{CPB}[4,28]$. Thus, improving the consumption of cement is the direct approach to increase the strength of $\mathrm{CPB}$. However, the cement/sand ratio should be controlled at a balanced value to save the cost of CPB. Similar findings were also obtained in the studies of Ercikdi [34] and Yilmaz and Ercikdi [35]. They remarked that the strength of $\mathrm{CPB}$ increased as the cement dosage increased (from 5 to 6 and 7 wt.\%) regardless of the tailings 


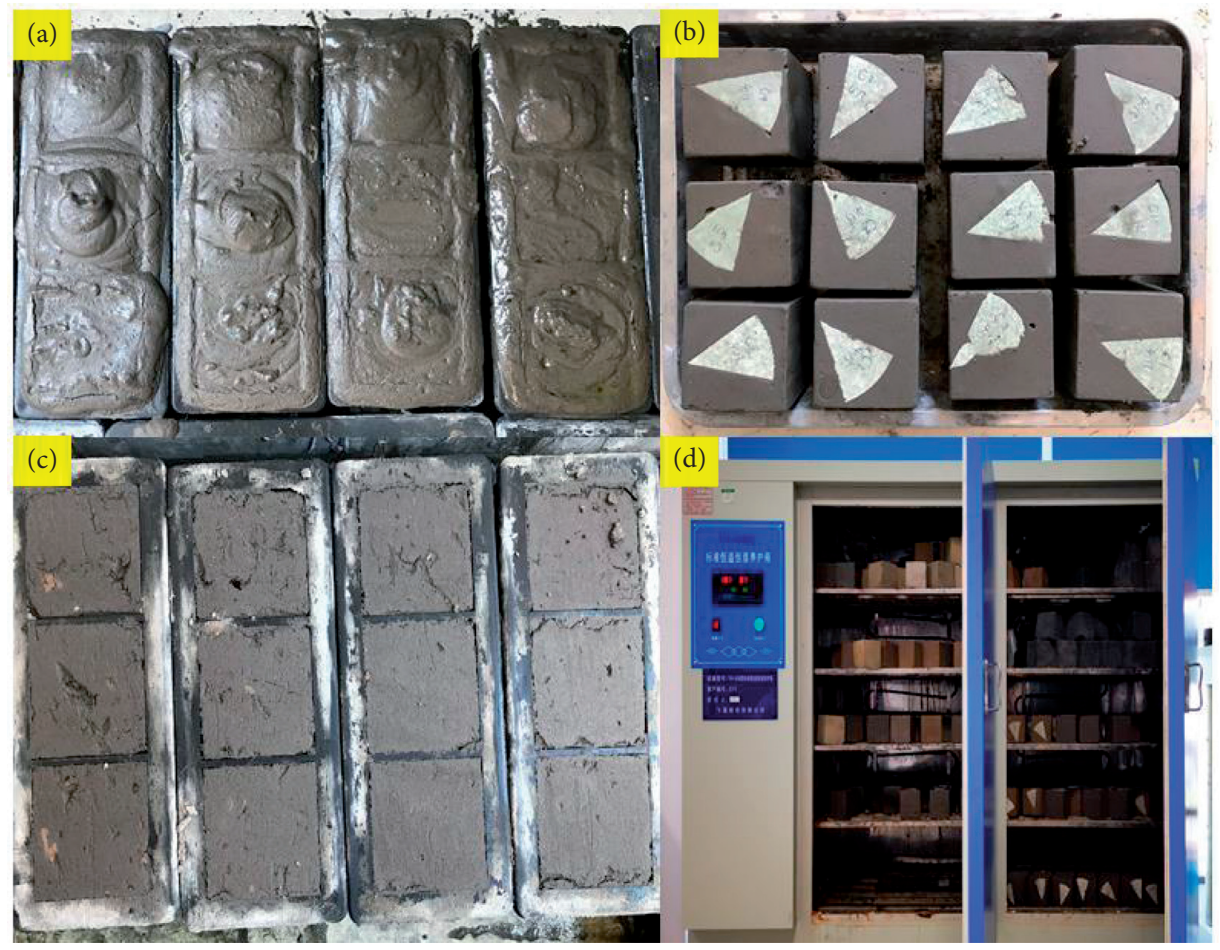

FIGURE 5: CPB specimens preparation and curing process.
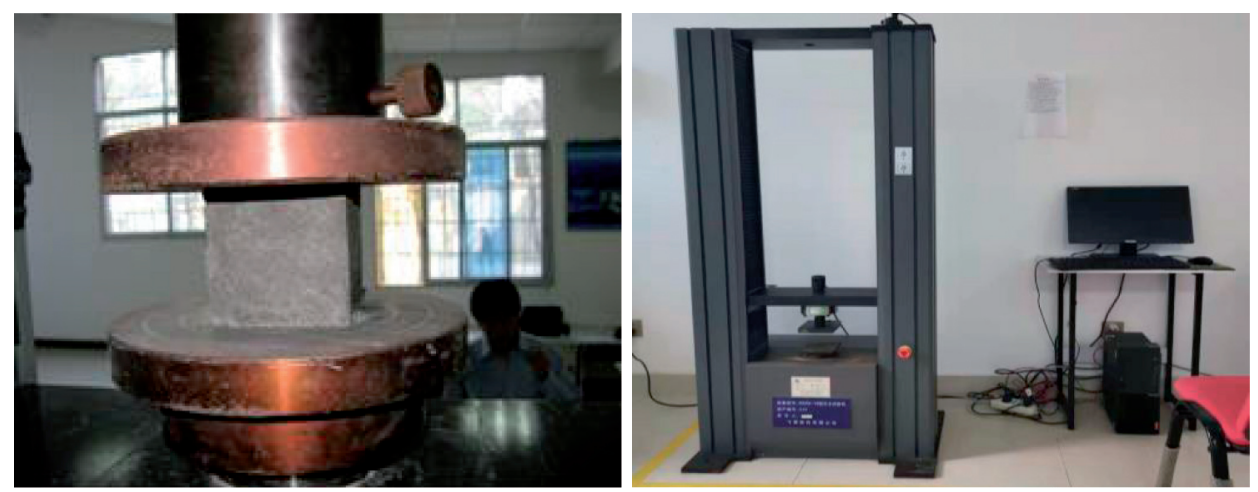

FIgURE 6: Laboratory UCS test.

type and curing periods. It was also manifested by the previous authors $[35,36]$ that the increase in the cement dosage had precious influence on the production of higher cementing bonds which in turn improved the strength gain.

Figure 11 presents the UCS values adding different cementitious materials, such as OPC, OPC +CG, and $\mathrm{OPC}+\mathrm{CFA}$, and the OPC is the control group. It can be seen that adding CG slightly improves the mechanical UCS, and the increments of $\mathrm{CPB}$ curing for 1 day, 3 days, and 28 days are $0.02 \mathrm{MPa}, 0.08 \mathrm{MPa}$, and $0.08 \mathrm{MPa}$. Thus, CG is not a suitable OPC substitute, which cannot improve the early strength, while adding CFA increases the UCS of CPB significantly, including the samples curing for 3 days and 28 days. Compared to the OPC group, the UCS increments of $\mathrm{OPC}+\mathrm{CFA}$ group samples curing for 3 days and 28 days are $0.34 \mathrm{MPa}$ and $4.67 \mathrm{MPa}$. It can be attributed to pozzolanic reaction occuring between CFA and calcium hydroxide
$\left(\mathrm{CaOH}_{2}\right)$ produced by OPC hydration [36, 37]. Furthermore, the previous researcher reported that the CFA has binding property as well as pozzolanic characteristic.

4.2. DNN Architecture Optimization. Figure 12 illustrates the update of the maximum correlation coefficient with GA iteration. It can be seen that the maximum correlation coefficient was 0.977 at the first iteration. The maximum correlation coefficient was increased to 0.979 at the seventh iteration and further increased to 0.989 at the $17^{\text {th }}$ iteration. After that, the maximum correlation coefficient remained the same until the $50^{\text {th }}$ iteration. The above results indicate that GA was efficient in the architecture tuning of DNN.

The optimum DNN architecture was determined after the GA iteration. The number of neurons was 95, 72, 158, 44, and 169 for the first to fifth hidden layer, respectively. 


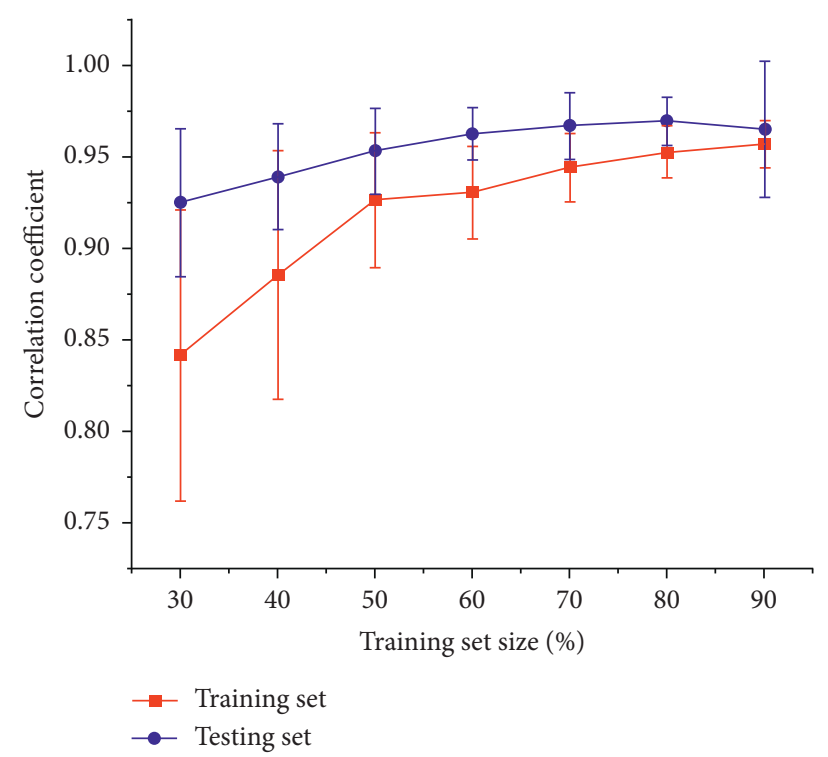

FIGURE 7: Influence of training set size on the performance of ANN modelling.

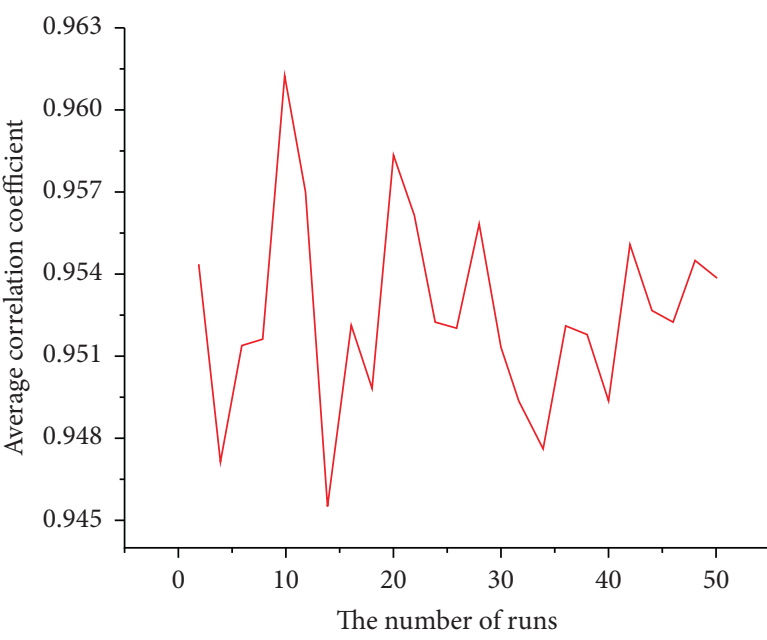

(a)

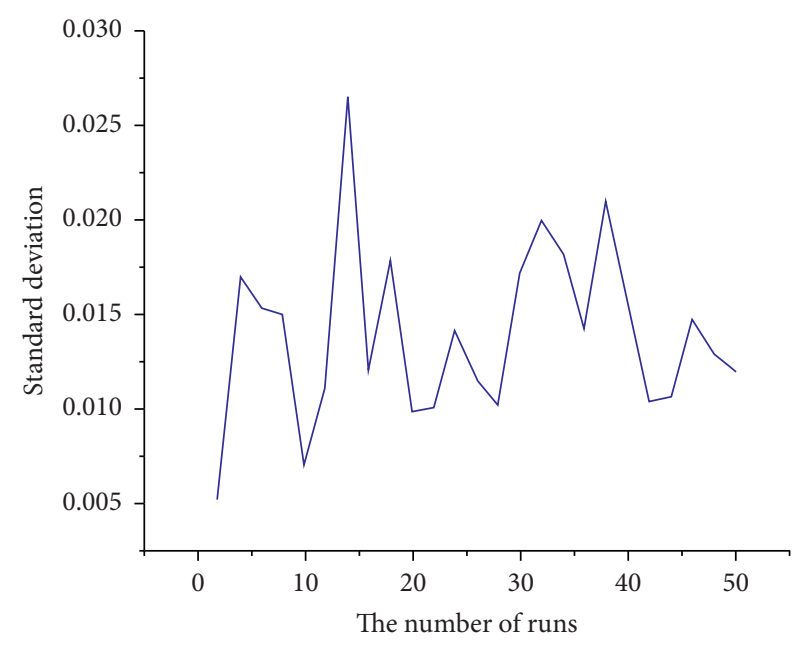

(b)

FIGURE 8: Influence of the number of runs on the average correlation coefficient and its standard deviation.

Considering the input layer and the output layer, the whole DNN architecture is illustrated in Figure 13.

4.3. DNN Prediction of UCS. In this section, the performance of the optimum DNN model is analyzed. Figure 14 illustrates the performance of the optimum DNN model on the training set and the testing set. As shown, the optimum DNN model achieved an average correlation coefficient of 0.97 on the training set and an average correlation coefficient of 0.99 on the testing set. The slight performance improvement on the testing set is due to the calculation difference on the training set and the testing set. As indicated before, the training performance was obtained by 5 -fold cross-validation and the testing performance was obtained after the training of the DNN using the whole training set.
The better performance on the testing set has been well documented in the literature, such as in [19].

Moreover, the standard deviation of the correlation coefficients was 0.0112 on the training set and 0.0065 on the testing set. Thus, the performance on the testing set was more stable than that on the training set.

Figure 15 presents a visualization of the performance of the optimum DNN model on the whole dataset. It can be seen that the predicted UCS agreed well with the experimental UCS, especially the changing trend. Moreover, the optimum DNN model could achieve good performance on CPB samples with high UCS values, as shown around the right part of Figure 7 . The above results further imply the robustness of the DNN model and its feasibility of the DNN prediction. 


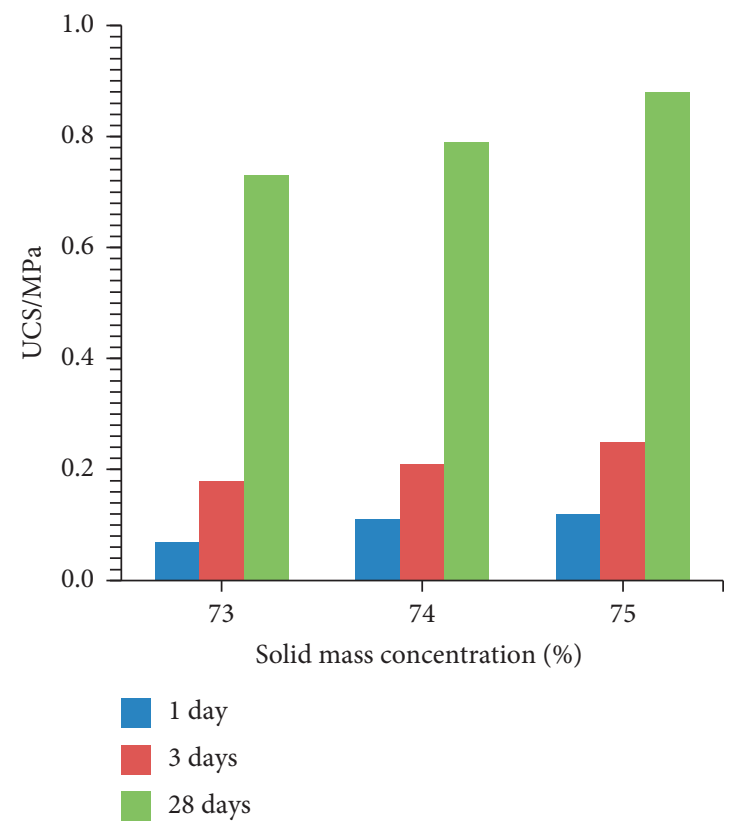

FIGURE 9: The results of UCS of CPB with cement/sand ratio of $1: 8$.

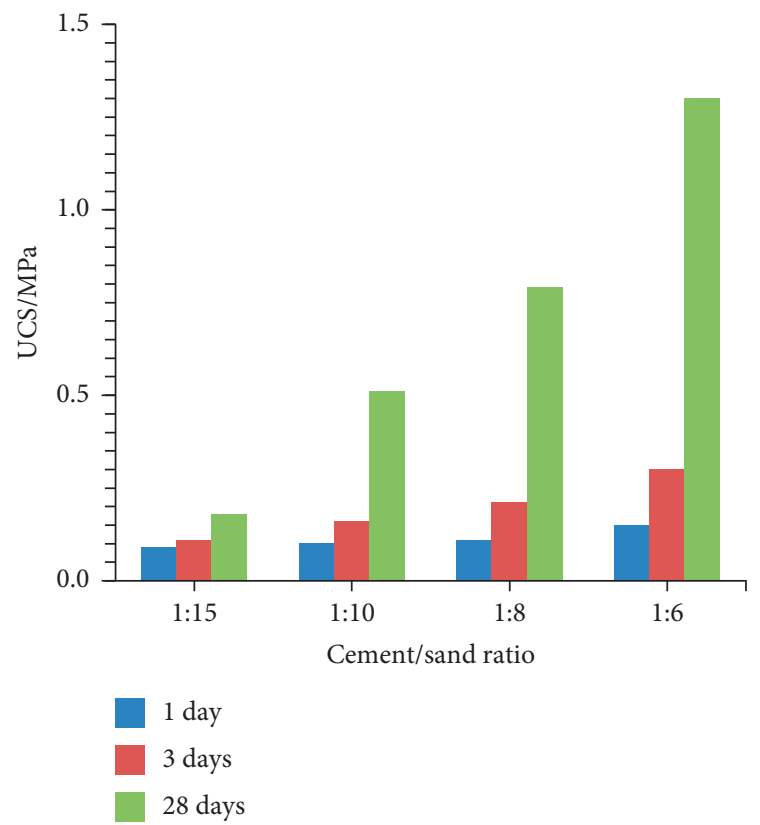

FIGURE 10: The results of UCS of CPB with solid mass concentration of $74 \%$.

4.4. Partial Dependence Plots and Importance Analysis. In this work, the partial dependence plots and importance score (IS) were used to investigate the influence of each input variable to the UCS of CPB. Partial dependence plots and IS are two important methods for the variable analysis and a detailed explanation of these two methods has been provided in [19]. The authors note that the summation of IS values was normalized to one for comparison purpose. Figure 16 illustrates the partial dependence plots and IS for each input variable.

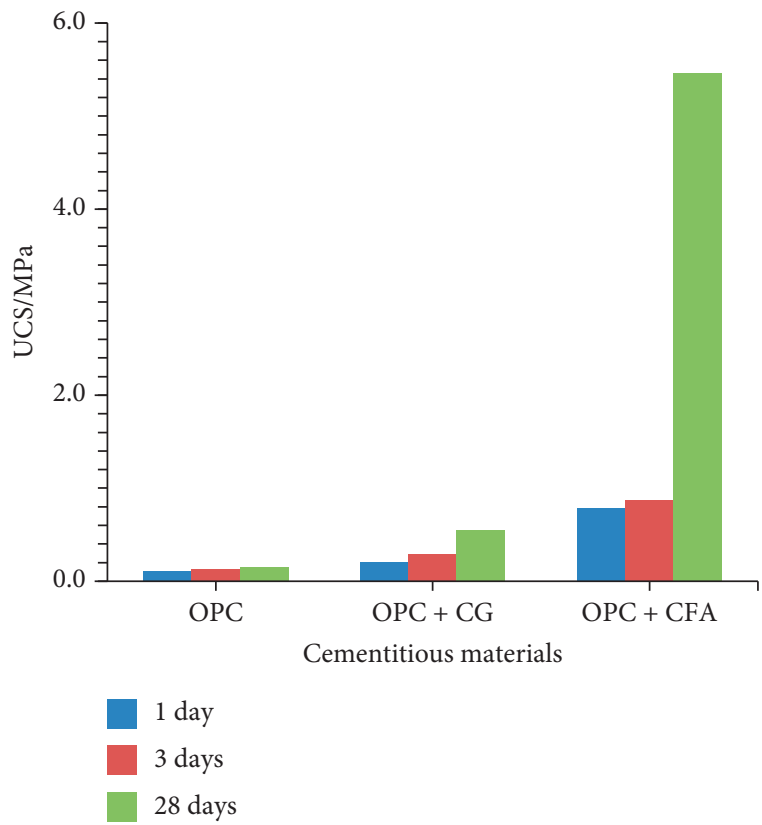

FIGURE 11: The results of UCS of CPB adding different cementitious materials.

It can be found that the UCS had an overall positive correlation with the OPC proportion, the CFA proportion, the solids content, and the curing time. A negative correlation was observed between the silt proportion and the UCS while the UCS had little correlation with the CG proportion. Based on the partial dependence plots, curing time was the most significant variable for UCS, followed by the CFA proportion. The CG was the least important variable for the CPB UCS.

The significance of each input variable to the UCS could be as easily indicated by the IS values. The IS value of the curing 


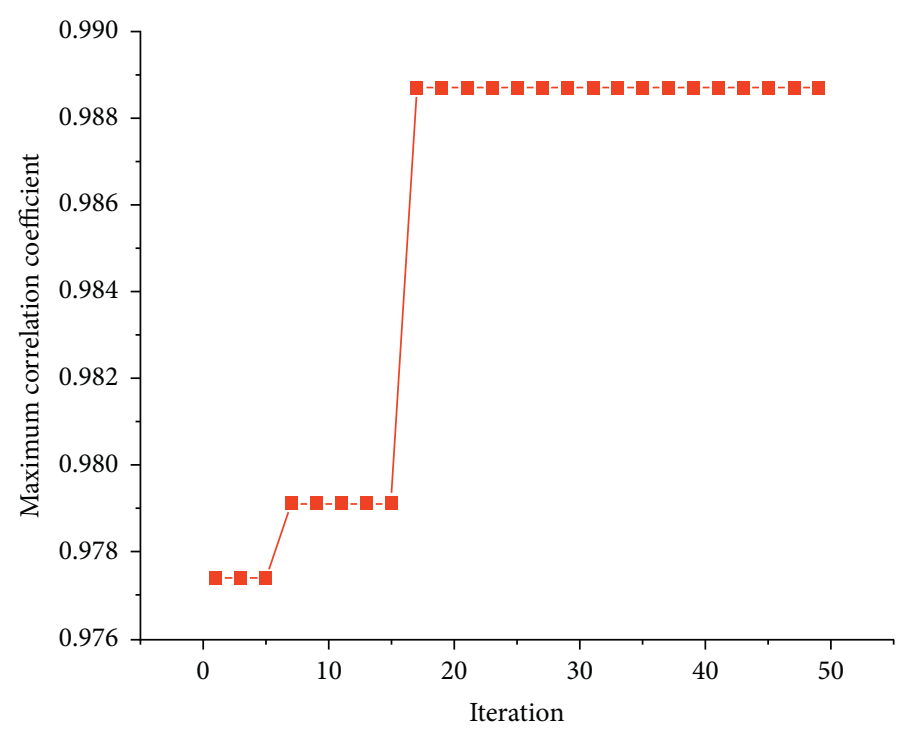

Figure 12: Update of the maximum correlation coefficient with GA iteration.

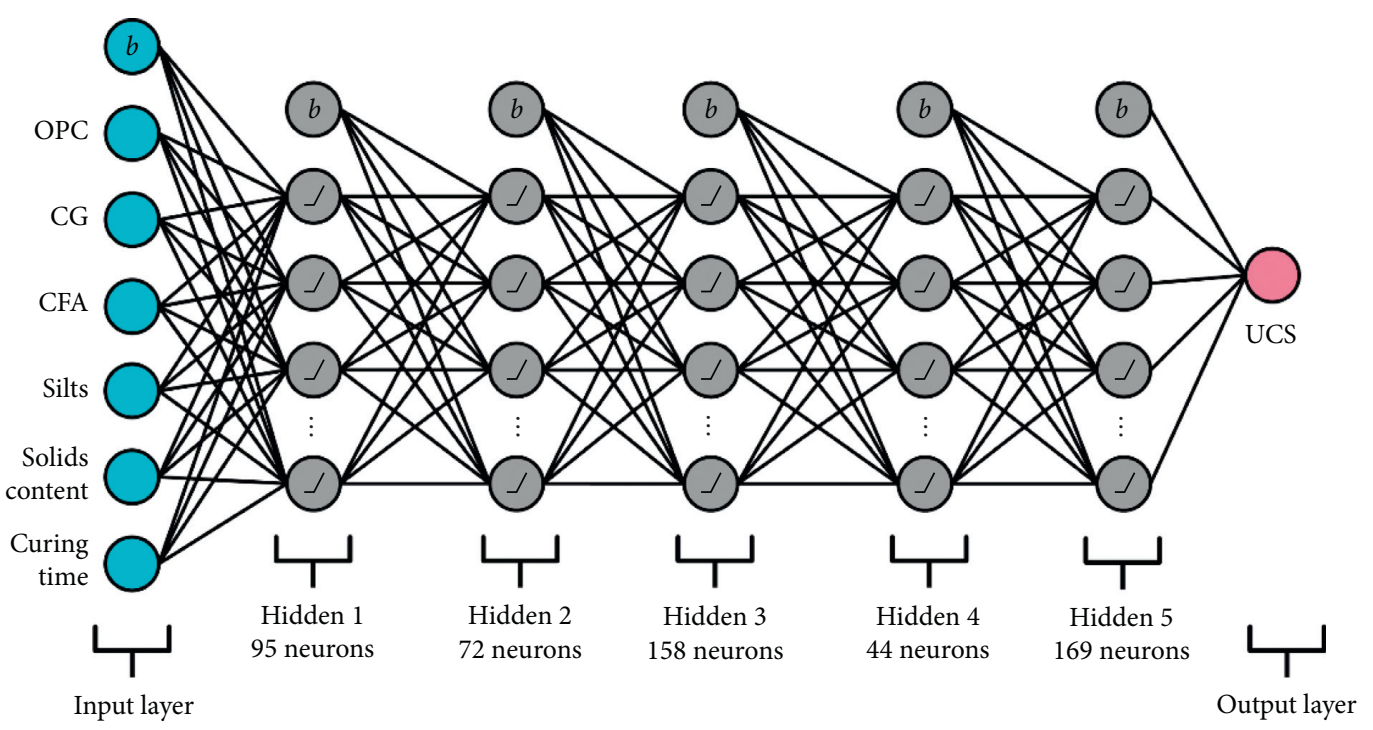

Figure 13: The optimum DNN architecture.

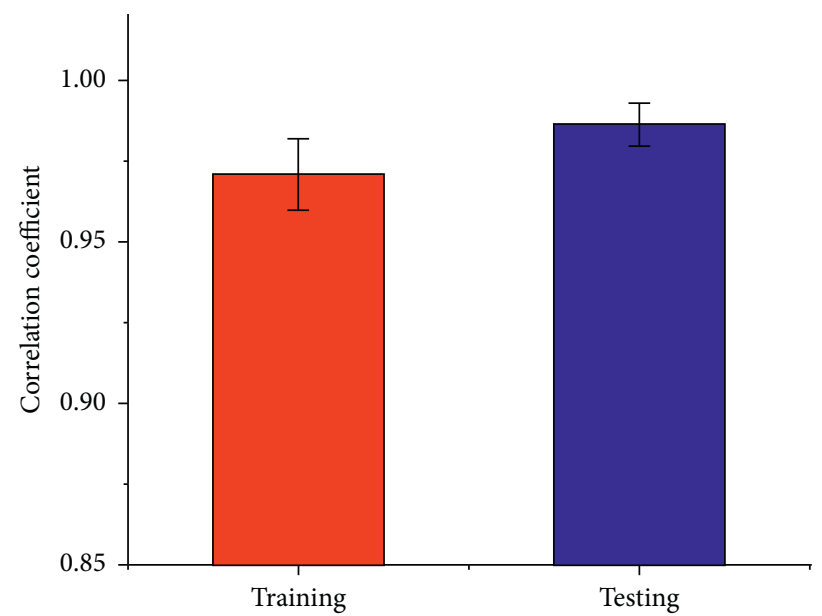

Figure 14: The performance of the optimum DNN model on the training set and the testing set. 


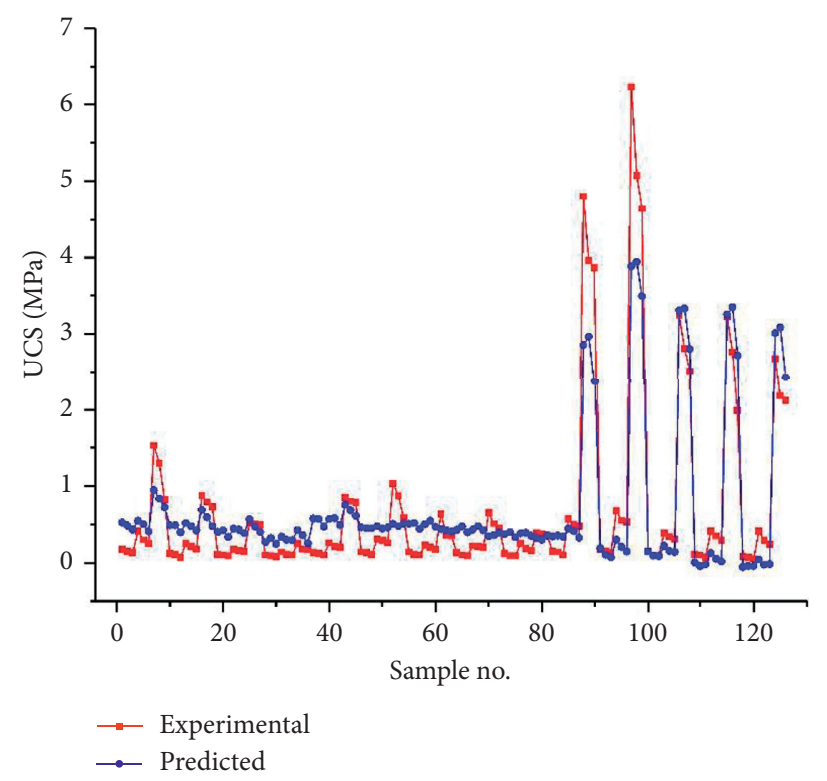

FIgURE 15: Performance visualization of the optimum DNN model on the whole dataset.

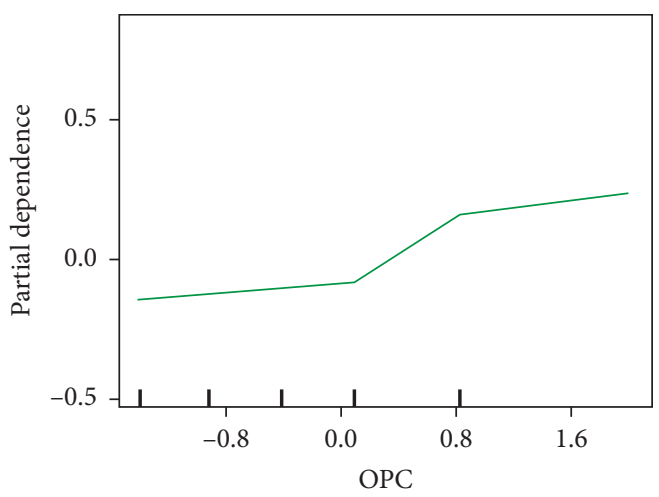

(a)

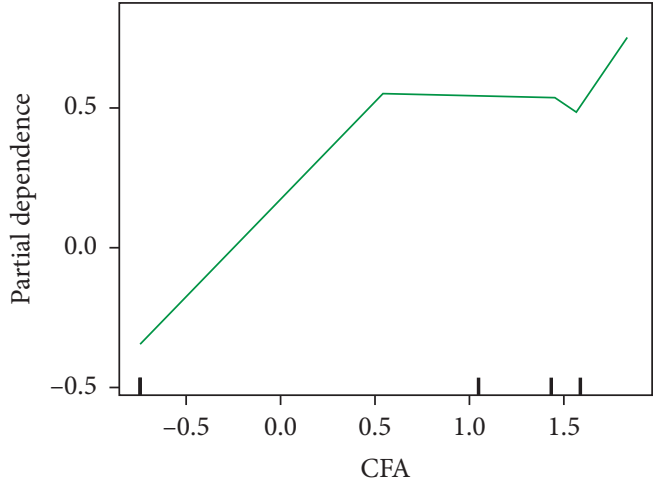

(c)

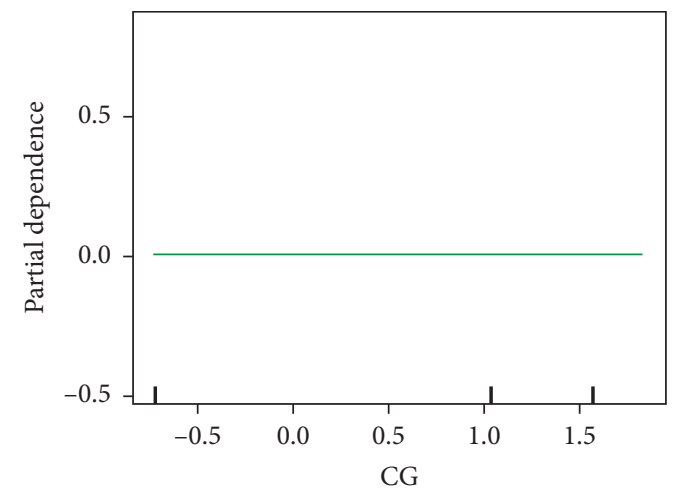

(b)

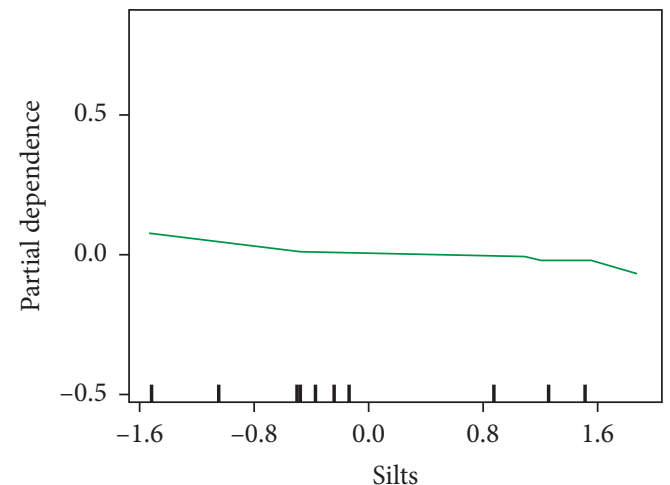

(d)

Figure 16: Continued. 


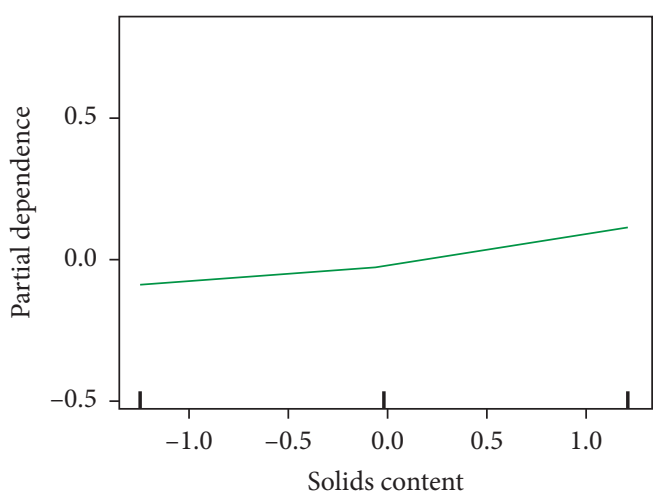

(e)

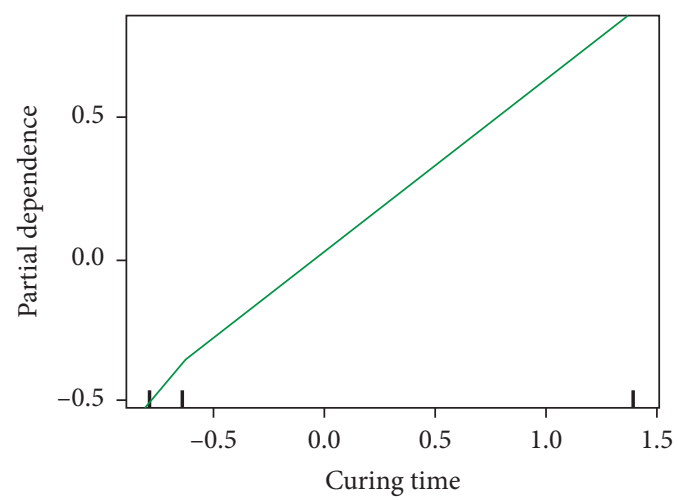

(f)

Figure 16: Partial dependence plots and IS values for each input variable. (a) IS $=0.085$. (b) IS $=0.011$. (c) IS $=0.257$. (d) IS $=0.045$. (e) IS $=0.198$. (f) IS $=0.404$.

time was found to be 0.404 , followed by 0.257 of the CFA proportion and 0.198 of the solids content. The IS values of the remaining three input variables were lower than 0.1 , which were 0.085 of the OPC proportion, 0.045 of the silt proportion, and 0.011 of the CG proportion. Generally speaking, the IS results agreed well with the partial dependence plots.

\section{Conclusions}

To facilitate the application of CPB in coal mines, the strength development of the silt-based CPB with different cementitious materials was investigated by lab experiments and DNN prediction. Based on the above results, the following conclusions can be drawn:

(1) The solid content, cement content (cement/sand ratio), and curing time present positive correlation with UCS. The CG can be used as a kind of OPC substitute, while adding CFA increases the UCS of CPB significantly.

(2) The convergence tests indicate the optimum training set size was $80 \%$ and the number of runs needs to be larger than 36 for the converged results.

(3) GA was efficient in the DNN architecture tuning with the maximum correlation coefficient being increased from 0.977 at the first iteration to 0.989 at the 17 th iteration. The optimum DNN architecture was characterized by $95,72,158,44$, and 169 within the first to fifth hidden layers.

(4) DNN was robust in predicting the UCS of CPB. The average correlation coefficient was 0.97 on the training set and was 0.99 on the testing set.

(5) Partial dependence plots indicate an overall positive correlation between the OPC proportion, the CFA proportion, the solids content, and the curing time with the UCS. In contrast, the silt proportion was negatively correlated with the UCS and CG proportion had little correlation with the UCS.

(6) The curing time was the most significant input variable for the UCS with an IS value of 0.404 , followed by 0.257 of the CFA proportion and 0.198 of the solids content. The CG proportion was the least significant input variable for the UCS with an IS value of 0.011 .

\section{Data Availability}

The data used to support the findings of this study are included within the article.

\section{Conflicts of Interest}

The authors declare that there are no conflicts of interest.

\section{Authors' Contributions}

C. X. and Q. C. conceived the project; C. X., Q. C, D. W., and Y. W. carried out experimental tests; X. W., C. X., and W. W. analyzed the experimental data. All authors discussed the results and commented on the manuscript.

\section{Acknowledgments}

The present research was financially supported by the State Key Laboratory of Safety and Health for Metal Mines (No. 2019-JSKSSYS-02) and Hunan Provincial Natural Science Foundation Project (No. 2020JJ5718).

\section{References}

[1] C.-C. Qi, "Big data management in the mining industry," International Journal of Minerals, Metallurgy and Materials, vol. 27, no. 2, pp. 131-139, 2020.

[2] G. Hilson, "Pollution prevention and cleaner production in the mining industry: an analysis of current issues," Journal of Cleaner Production, vol. 8, no. 2, pp. 119-126, 2000.

[3] W. Zhou, X. Shi, X. Lu, C. Qi, B. Luan, and F. Liu, "The mechanical and microstructural properties of refuse mudstone-GGBS-red mud based geopolymer composites made with sand," Construction and Building Materials, vol. 253, Article ID 119193, 2020.

[4] Q. Chen, Q. Zhang, C. Qi, A. Fourie, and C. Xiao, "Recycling phosphogypsum and construction demolition waste for cemented paste backfill and its environmental impact," Journal of Cleaner Production, vol. 186, pp. 418-429, 2018. 
[5] Q. Chen, Q. Zhang, A. Fourie, and C. Xin, "Utilization of phosphogypsum and phosphate tailings for cemented paste backfill," Journal of Environmental Management, vol. 201, pp. 19-27, 2017.

[6] X. Chen, X. Z. Shi, S. Zhang et al., "Fiber-Reinforced cemented paste backfill: the effect of fiber on strength properties and estimation of strength using nonlinear models," Materials, vol. 13, 2020.

[7] C. Qi and A. Fourie, "Cemented paste backfill for mineral tailings management: review and future perspectives," Minerals Engineering, vol. 144, Article ID 106025, 2019.

[8] Z. Su, Q. S. Chen, Q. L. Zhang, and D. M. Zhang, "Recycling lead-zinc tailings for cemented paste backfill and stabilisation of excessive metal," Minerals, vol. 9, no. 11, 2019.

[9] A. W. L. Dudeney, B. K. C. Chan, S. Bouzalakos, and J. L. Huisman, "Management of waste and wastewater from mineral industry processes, especially leaching of sulphide resources: state of the art," International Journal of Mining, Reclamation and Environment, vol. 27, no. 1, pp. 2-37, 2013.

[10] S. Cao, W. Song, and E. Yilmaz, "Influence of structural factors on uniaxial compressive strength of cemented tailings backfill," Construction and Building Materials, vol. 174, pp. 190-201, 2018.

[11] X. Deng, B. Klein, L. Tong, and B. De Wit, "Experimental study on the rheological behavior of ultra-fine cemented backfill," Construction and Building Materials, vol. 158, pp. 985-994, 2018.

[12] H. Lu, C. Qi, C. Li, D. Gan, Y. Du, and S. Li, “A light barricade for tailings recycling as cemented paste backfill," Journal of Cleaner Production, vol. 247, Article ID 119388, 2020.

[13] L. Liu, Z. Fang, M. Wang, C. Qi, Y. Zhao, and C. Huan, "Experimental and numerical study on rheological properties of ice-containing cement paste backfill slurry," Powder Technology, vol. 370, pp. 206-214, 2020.

[14] C. Qi and A. Fourie, "Numerical investigation of the stress distribution in backfilled stopes considering creep behaviour of rock mass," Rock Mechanics and Rock Engineering, vol. 52, no. 9, pp. 3353-3371, 2019.

[15] D. Ouattara, T. Belem, M. Mbonimpa, and A. Yahia, "Effect of superplasticizers on the consistency and unconfined compressive strength of cemented paste backfills," Construction and Building Materials, vol. 181, pp. 59-72, 2018.

[16] T. Yllmaz, B. Ercikdi, and H. Deveci, "Utilisation of construction and demolition waste as cemented paste backfill material for underground mine openings," Journal of Environmental Management, vol. 222, pp. 250-259, 2018.

[17] F. Cihangir, B. Ercikdi, A. Kesimal, S. Ocak, and Y. Akyol, "Effect of sodium-silicate activated slag at different silicate modulus on the strength and microstructural properties of full and coarse sulphidic tailings paste backfill," Construction and Building Materials, vol. 185, pp. 555-566, 2018.

[18] C. Qi, A. Fourie, and Q. Chen, "Neural network and particle swarm optimization for predicting the unconfined compressive strength of cemented paste backfill," Construction and Building Materials, vol. 159, pp. 473-478, 2018.

[19] C. Qi, A. Fourie, Q. Chen, and Q. Zhang, “A strength prediction model using artificial intelligence for recycling waste tailings as cemented paste backfill," Journal of Cleaner Production, vol. 183, pp. 566-578, 2018.

[20] C. Qi, Q. Chen, A. Fourie, and Q. Zhang, "An intelligent modelling framework for mechanical properties of cemented paste backfill," Minerals Engineering, vol. 123, pp. 16-27, 2018.

[21] J. Qiu, Z. Guo, L. Li, S. Zhang, Y. Zhao, and Z. Ma, "A hybrid artificial intelligence model for predicting the strength of foam-cemented paste backfill," IEEE Access, vol. 8, pp. 84569-84583, 2020.

[22] E. Li, J. Zhou, X. Shi et al., "Developing a hybrid model of salp swarm algorithm-based support vector machine to predict the strength of fiber-reinforced cemented paste backfill," Engineering with Computers, vol. 2020, 2020.

[23] Y. Sun, G. Li, J. Zhang, J. Sun, and J. Xu, "Development of an ensemble intelligent model for assessing the strength of cemented paste backfill," Advances in Civil Engineering, vol. 2020, Article ID 1643529, 6 pages, 2020.

[24] Y. Huang, J. Zhang, Q. Zhang, and S. Nie, "Backfilling technology of substituting waste and fly ash for coal underground in China coal mining area," Environmental Engineering and Management Journal, vol. 10, no. 6, pp. 769-775, 2011.

[25] J. Zhang, Q. Zhang, Q. Sun, R. Gao, D. Germain, and S. Abro, "Surface subsidence control theory and application to backfill coal mining technology," Environmental Earth Sciences, vol. 74, no. 2, pp. 1439-1448, 2015.

[26] D. Wang, Q. Zhang, Q. Chen, C. Qi, Y. Feng, and C. Xiao, "Temperature variation characteristics in flocculation settlement of tailings and its mechanism," International Journal of Minerals, Metallurgy and Materials, vol. 21, pp. 1-11, 2020.

[27] Y. He, Q. S. Chen, C. C. Qi, Q. L. Zhang, and C. C. Xiao, "Lithium slag and fly ash-based binder for cemented fine tailings backfill," Journal of Environmental Management, vol. 248, Article ID 109282, 2019.

[28] Q.-S. Chen, Q.-L. Zhang, A. Fourie, X. Chen, and C.-C. Qi, "Experimental investigation on the strength characteristics of cement paste backfill in a similar stope model and its mechanism," Construction and Building Materials, vol. 154, pp. 34-43, 2017.

[29] M. Kuhn and K. Johnson, Applied Predictive Modeling, Springer-Verlag, New York, NY, USA, 2013.

[30] J. Tian, C. Qi, Y. Sun, Z. M. Yaseen, and B. T. Pham, "Permeability prediction of porous media using a combination of computational fluid dynamics and hybrid machine learning methods," Engineering with Computers, vol. 2020, 2020.

[31] F. Pedregosa, G. Varoquaux, A. Gramfort et al., "Scikit-learn: machine learning in Python," Journal of Machine Learning Research, vol. 12, pp. 2825-2830, 2011.

[32] J. Grefenstette, "Optimization of control parameters for genetic algorithms," IEEE Transactions on Systems, Man, and Cybernetics, vol. 16, no. 1, pp. 122-128, 1986.

[33] Y. K. Liu, Q. L. Zhang, Q. S. Chen, C. C. Qi, Z. Su, and Z. D. Huang, "Utilisation of water-washing pre-treated phosphogypsum for cemented paste backfill," Minerals, vol. 9, no. 3, 175 pages, 2019.

[34] B. Ercikdi, T. Yılmaz, and G. Külekci, "Strength and ultrasonic properties of cemented paste backfill," Ultrasonics, vol. 54, no. 1, pp. 195-204, 2014.

[35] T. Yllmaz and B. Ercikdi, "Predicting the uniaxial compressive strength of cemented paste backfill from ultrasonic pulse velocity test," Nondestructive Testing and Evaluation, vol. 31, no. 3, pp. 247-266, 2015.

[36] X. Chen, X. Z. Shi, J. Zhou, Z. Yu, and P. S. Huang, "Determination of mechanical, flowability, and microstructural properties of cemented tailings backfill containing rice straw," Construction and Building Materials, vol. 246, Article ID 118520, 2020.

[37] Y. Feng, Q. S. Chen, Y. L. Zhou et al., "Modification of glass structure via $\mathrm{CaO}$ addition in granulated copper slag to enhance its pozzolanic activity," Construction and Building Materials, vol. 240, Article ID 117970, 2020. 